\title{
A CONCEPT OF THE DIFFERENTIALLY DRIVEN THREE WHEELED ROBOT
}

\author{
M. KELEMEN*, D.J. COLVILLE, T. KELEMENOVÁ \\ I. VIRGALA and L. MIKOVÁ \\ Technical University of Košice, Faculty of Mechanical Engineering \\ Kosice, SLOVAK REPUBLIC \\ E-mail: michal.kelemen@tuke.sk
}

\begin{abstract}
The paper deals with the concept of a differentially driven three wheeled robot. The main task for the robot is to follow the navigation black line on white ground. The robot also contains anti-collision sensors for avoiding obstacles on track. Students learn how to deal with signals from sensors and how to control DC motors. Students work with the controller and develop the locomotion algorithm and can attend a competition.
\end{abstract}

Key words: robot, education, wheeled locomotion, sensor, mechatronics.

\section{Introduction}

A differentially driven three wheeled robot or line follower robot (Fig.1) has been developed at the Technical University of Kosice, Faculty of Mechanical Engineering. It is designed in accordance with the competition rules from the ISTROBOT competition organized at STU in Bratislava. Students have a possibility to try working with navigation sensors, to drive actuators, design algorithms, etc.

The design of the robot is based on a three wheeled undercarriage with two differentially driven wheels and one castor wheel. This design offers very good manoeuvre abilities of the robot on a small area. The robot has to follow the navigation line (black colour) on white ground. The robot has to do also other activities as avoiding obstacles (brick), opening a door at the tunnel and passing through the tunnel, passing up and down the ramp and passing through the junction and line interruption (Figs 2, 3, 4) (Colville, 2012; Jurisici et al., 2005; Kacir, 2011; Mikova, 2011).

Locomotion uphilland downhill on the bridge changes the DC motor loading. It is necessary to apply a regulation process for securing better locomotion. The robot uses the line recognition sensors for line sensing. All the above mentioned situations can change sensing conditions for the line sensors (change of distance between the line and sensor, change of lighting condition, etc.). For these reasons, the line sensing is not so trivial. The robot has to avoid obstacle if any object occurs on the race. The sensing loop in the control algorithm has to be very fast. The robot uses the combination of the contactless optical sensors and tactile sensors for safe obstacle avoidance. The strategy of the robot behaviour is also important. The situation is more complicated because an optimized control program with minimum steps is required for obtaining fast locomotion.

Educational process supported with practical exercises helps students to improve in various fields of study. The proposed didactic model supports the applied educational approach by solving the defined problem situation. This approach, first of all, applies the problem interpretation-method and heuristic and research methods. Problem solving philosophy of education is based on the fact that during a problem task solving and also by the model situation problem solving processes the students acquire experience from their creative activity and they creatively master their knowledge and the ways of their activity. The students join a problem searching and problem solving processes through the activities of the teacher. This way the

\footnotetext{
* To whom correspondence should be addressed
} 
students learn how to learn and acquire knowledge independently and in the process they apply their background knowledge and along with it they are personally experienced from their creative activities. The practical realization of the task and the personal involvement in the problem solving imitate the team work pattern and the individuals may be aware of their importance within it. This approach is also of psychological and social importance

Creative thinking is developed during problem solving when the student has some difficulty in his activity, some conflict, when he encounters something unknown, surprising and incomprehensible, and he does not know the way of overcoming the problem or the obstruction and he can not solve it on the basis of his actual knowledge. In other words, the mechatronics is not only about the lectures and it has to pass directives to the students hands. So that the hands may act, they should be directed by the brain. The brain has to think what directive to get out and what activities will be performed by the hands. If something is coming to the student's hands he means the integral chain of consideration and researching and the individual study and consultations among the team members and also with the pedagogue who is both the guardian and the anchorman of the entire project. In this manner the students are learning the philosophy of the mechatronics. Their thinking and creativity get a new dimensions which enables the student's capability to control better problem solving situations in practice. And graduates trained in this manner markedly increase the probability of being successful in their practice. At the same time they may contribute to the competitiveness of their employers (Nitulescu, 2008; Vitko et al., 2010; Maniarski and Kuryło, 2006).
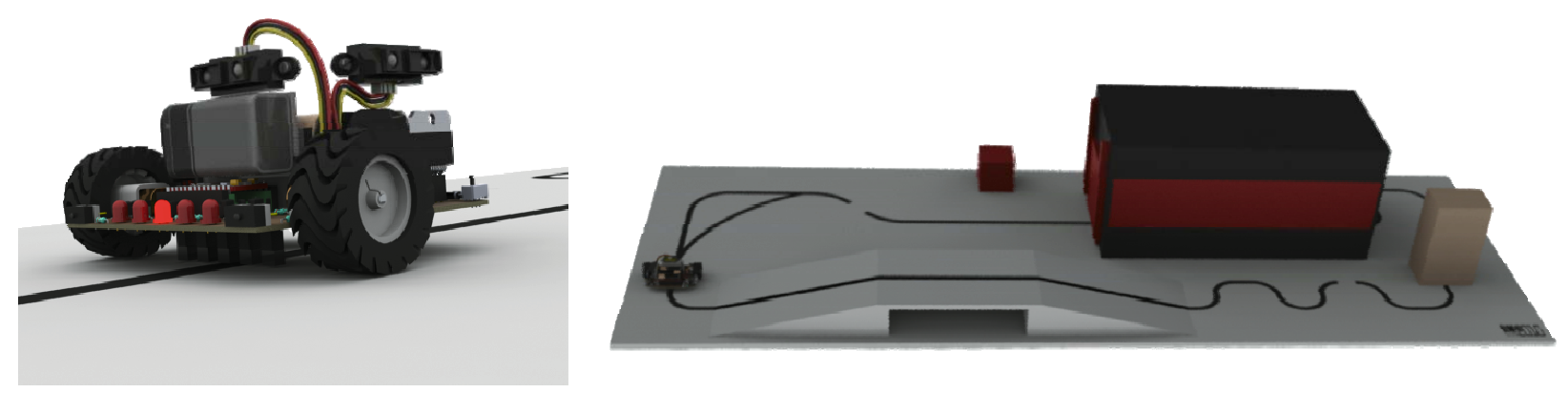

Fig.1. Line follower robot with competition race.
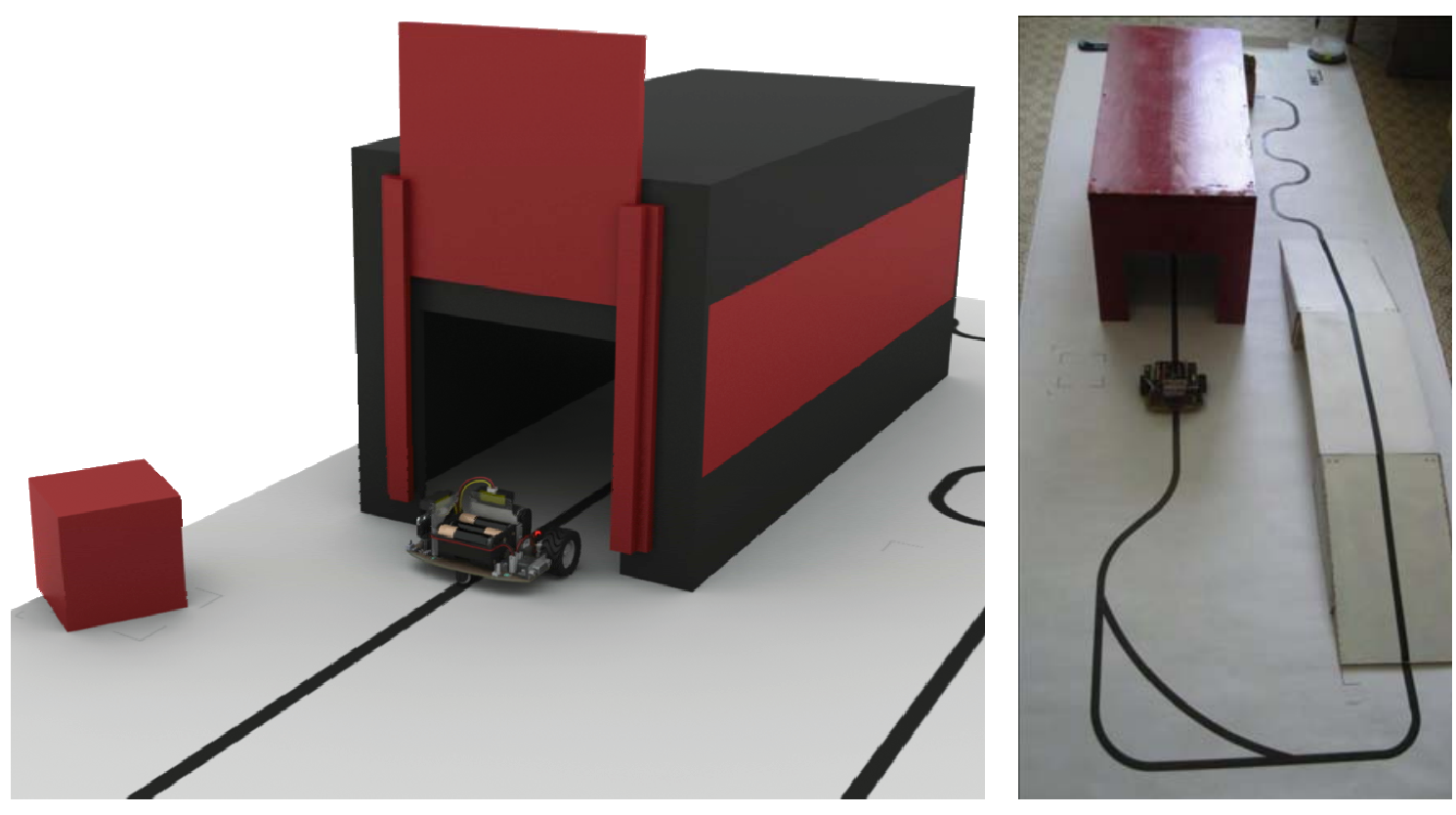

Fig.2. Red cube placement and opened tunnel. 


\section{Functions and tasks of the robot}

The robot has to pass over a tunnel (Fig.2). The aim of the tunnel is to change the optical condition for line recognition. The tunnel is closed with a door and the robot has to open this door. The robot needs to locate a $10 \mathrm{~cm}$ red cube to the left of the track ( 15 to $20 \mathrm{~cm}$ from the line), and push it a minimum of $2 \mathrm{~cm}$ in any direction to open the door, return to the line and continue through the tunnel. Its weight is $250 \mathrm{~g}$. The door is automatically closed, when the robot passes through the tunnel. The robot will detect the tunnel door using a collision sensor, and then look to see if the cube is there using another collision sensor.

The robot has to 1 move up and down the ramp (Fig.3). The robot needs to detect that it is on an incline and speed up to have enough momentum to carry itself to the top of the ramp. It must also detect when it is going down the ramp in order to slow down, or it might lose control.

If any object is detected on the track, the robot has to avoid the obstacle (Fig.4). The robot needs to detect the obstacle (brick) placed in the path of the line, and determine whether or not it is the tunnel door or a plain obstacle, and then navigate around it. The robot needs to return to the line within $30 \mathrm{~cm}$ after the obstacle. It will detect the obstacle using the collision sensor (Colville, 2012; Kacir, 2011; Maniarski and Kuryło, 2006).
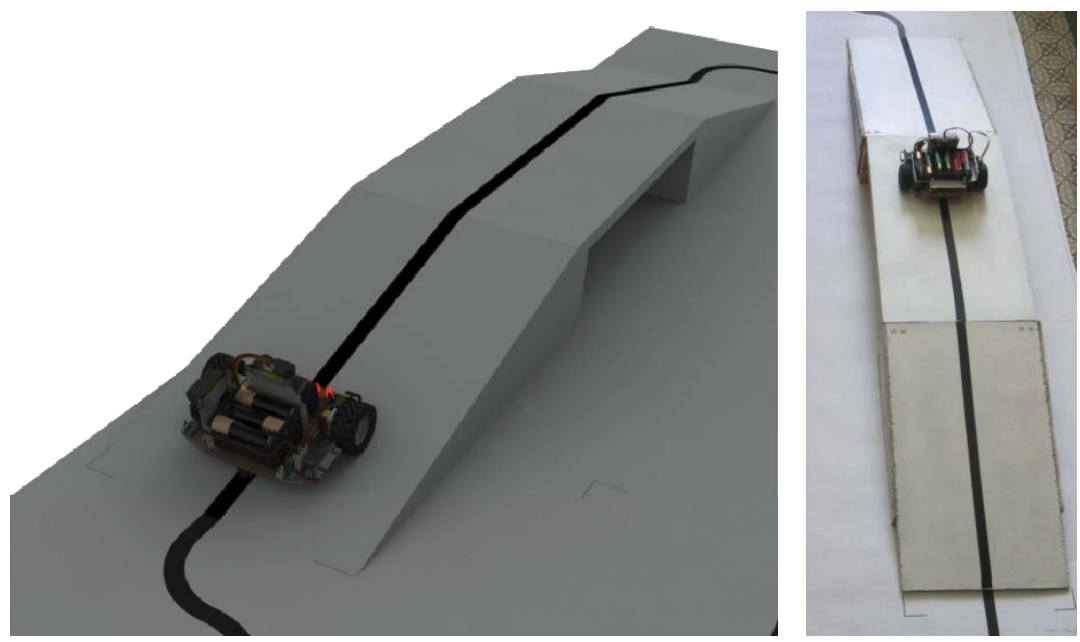

Fig.3. Robot LINA 2010 pass-over the ramp.
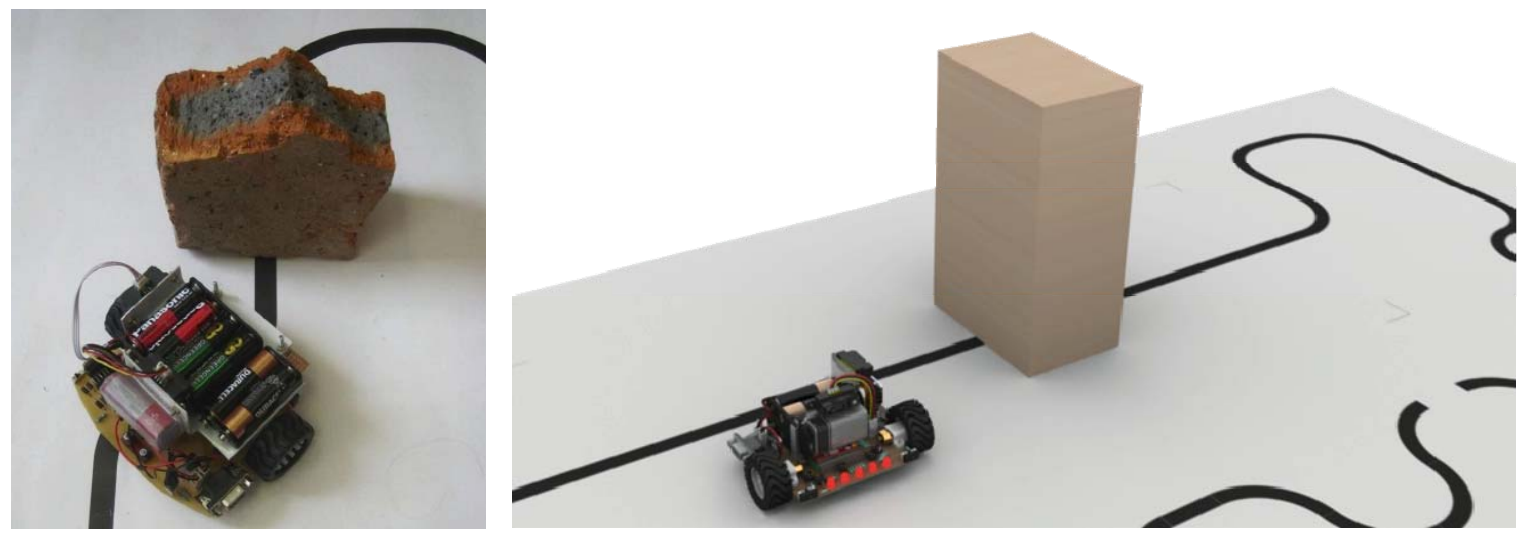

Fig.4. Obstacle (brick) on the track. 


\section{The control unit}

In order to control the robot we used the Basic Atom Pro 28-M module (Fig.5) with the icrocontroller Hitachi HD64F3694GFYV processor which uses 32 Bit floating Point math with over 100,000 BASIC instructions per second.

The module includes $32 \mathrm{~KB}$ of Program Space (FLASH), $2 \mathrm{~KB}$ of User Memory (RAM) and $4 \mathrm{~KB}$ of User Data Storage (EEPROM). This module has 28 pins, of which 20 can be programmed as either IN or OUTPUT. Some of the pins have special features such as ADIN (Analogue to Digital Input with resolution 16bit) which we will be utilising on the QRD sensors and the Sharp sensors. The module also enables the use of 3 hardware Timers, 3 hardware PWM I/O Pins, hardware interrupts, 8 analog-to-Digital Capable I/O pins, 16 servo background control. In order to communicate with the module we used a built-in RS232 connection. The module can be programmed using the BASIC language, C or ASM. Our students used the BASIC language, because of its simplicity. The robot is controlled via using the logical functions designed as structured flow charts rewritten into the Basic language.

The robot has no frame. All parts including the DC motors are placed on a PCB (printed circuit board) (Fig.5). DC motors are controlled via a simple PD controller algorithm. The DC motors are controlled using a H-bridge, which separates the low voltage, low current signal from the microcontroller and the high voltage, high current supply which the DC motors require. Both of the DC motors vary slightly, they have gear heads inside them with slightly different friction rates, calibrating of both the motors would be possible but since the robot is constantly making adjustments it would not really be required. The H-bridge allows the motor to change direction by changing the polarity of voltage across the motors terminals.

The five LEDs are only an addition to help calibrate each of the reflective light sensor, and to indicate that each of the sensor is functioning correctly (Colville, 2012; Jurisica et al., 2005; Kacir, 2011).
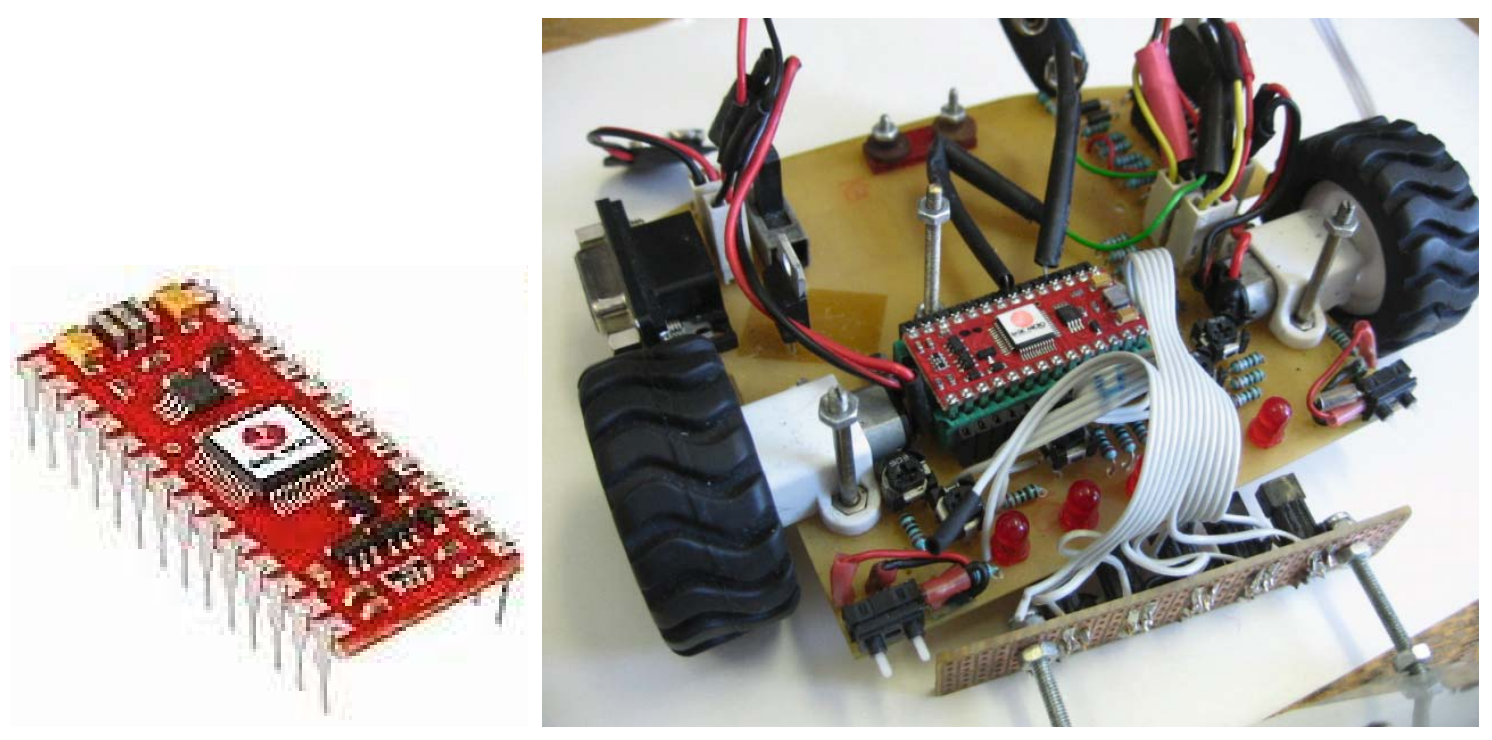

Fig.5. Basic Atom Pro 28-M microcontroller and PCB with controller.

\section{Testing of the line recognition sensors}

\subsection{The Line Recognition sensor selection}

Three various types of reflective infrared sensors (QRD1114, LTH209-1, CNY70) were tested in order to choose a suitable line recognition sensor (Fig.6). All tested sensors are designed as infrared reflective optocoupler with daylight filter. All sensors were attached to the movable one-axis table with 
increment of $1 \mathrm{~mm}$ during testing with a white reflective target area. The distance of the sensors was changed in the range of $0-20 \mathrm{~mm}$. The collector current of each tested sensor was observed in the daylight, the halogen light and the dark environment. The aim of this testing was to find, which sensor has minimum sensitivity to changed light intensity of the environment (Colville, 2012).

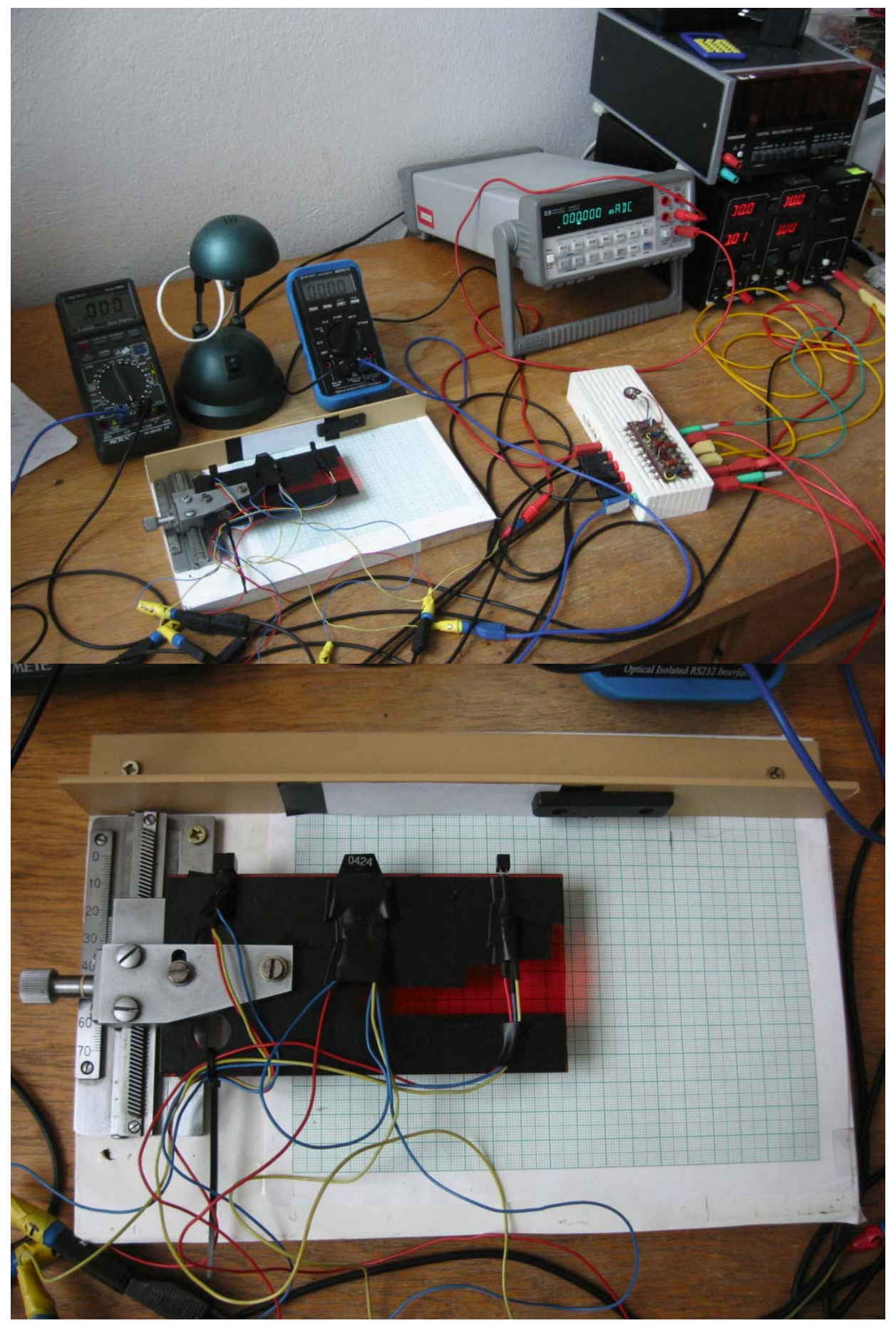

Fig.6. Testing of various type of the line recognition sensors. 
Figure 7 shows the results of testing. The QRD 1114 sensor has the best properties. The QRD 1114 has the largest collector current than other tested sensors. A useful range of distance for line recognition is $10 \mathrm{~mm}$. Consequently, the QRD 1114 was selected for the robot. In order to analyse the performance and characteristics of this sensor, the two different sensors QRD 1114 were tested using different surface colours, white and black. White and black were chosen as these are the two colours utilised in the competition for the line follower. The two different sensors were tested in order to find out the level of variation between sensors, and to check whether each of the sensor would require individual adjustment to gain the correct readings (Fig.8). The two sensors which were tested were from the same batch that had already been installed in the robot. In order to test the sensors a bench setup was created using the 1 axis table with increments of $1 \mathrm{~mm}$.

The graphs (Fig.8) show that both of the sensors give slightly different values for the same distances. In order to end up with similar results for both sensors on the line following robot, we will require adjustable potentiometer dividers, so we can change the value between the logical 1 and the logical 0.
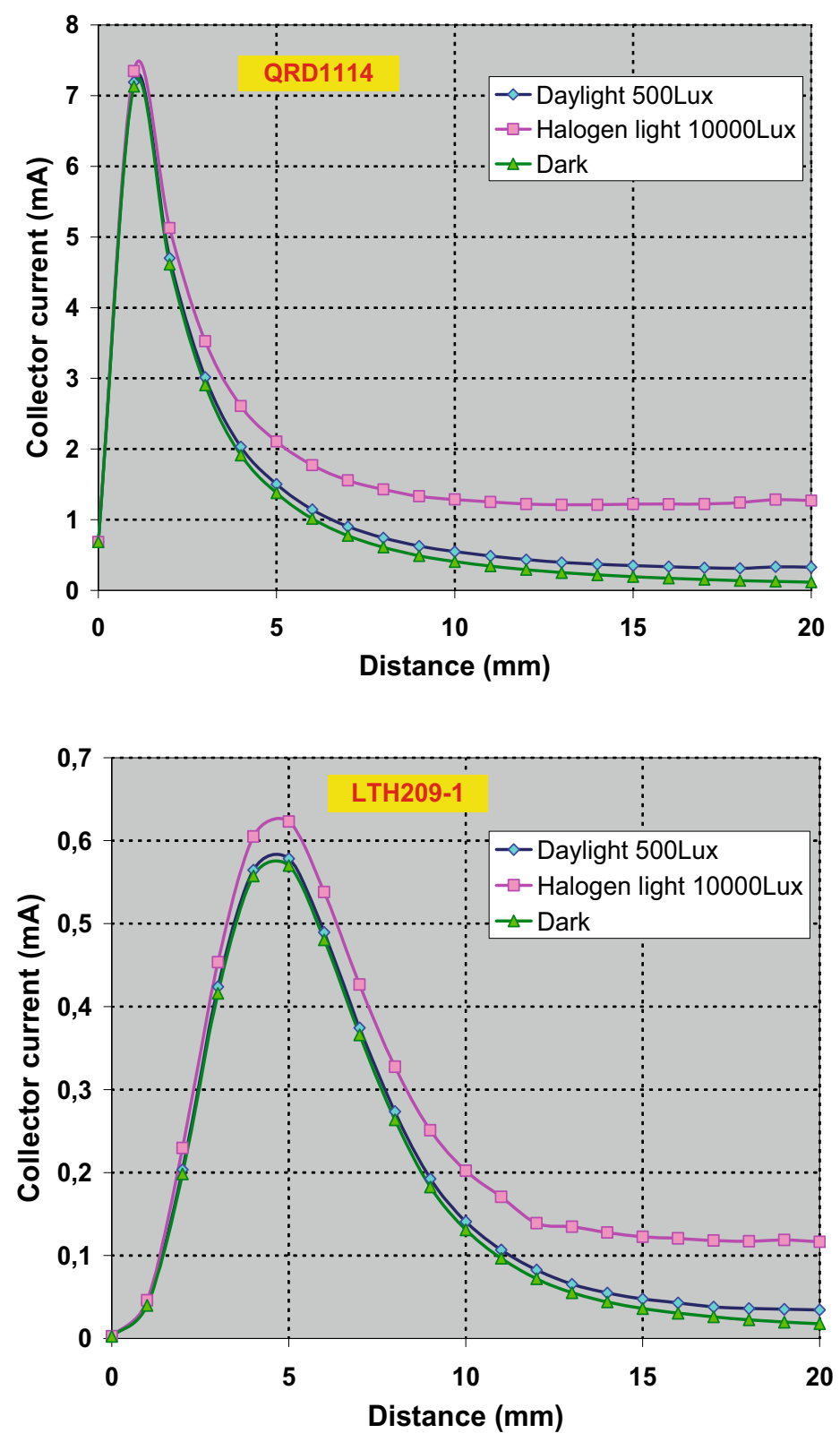

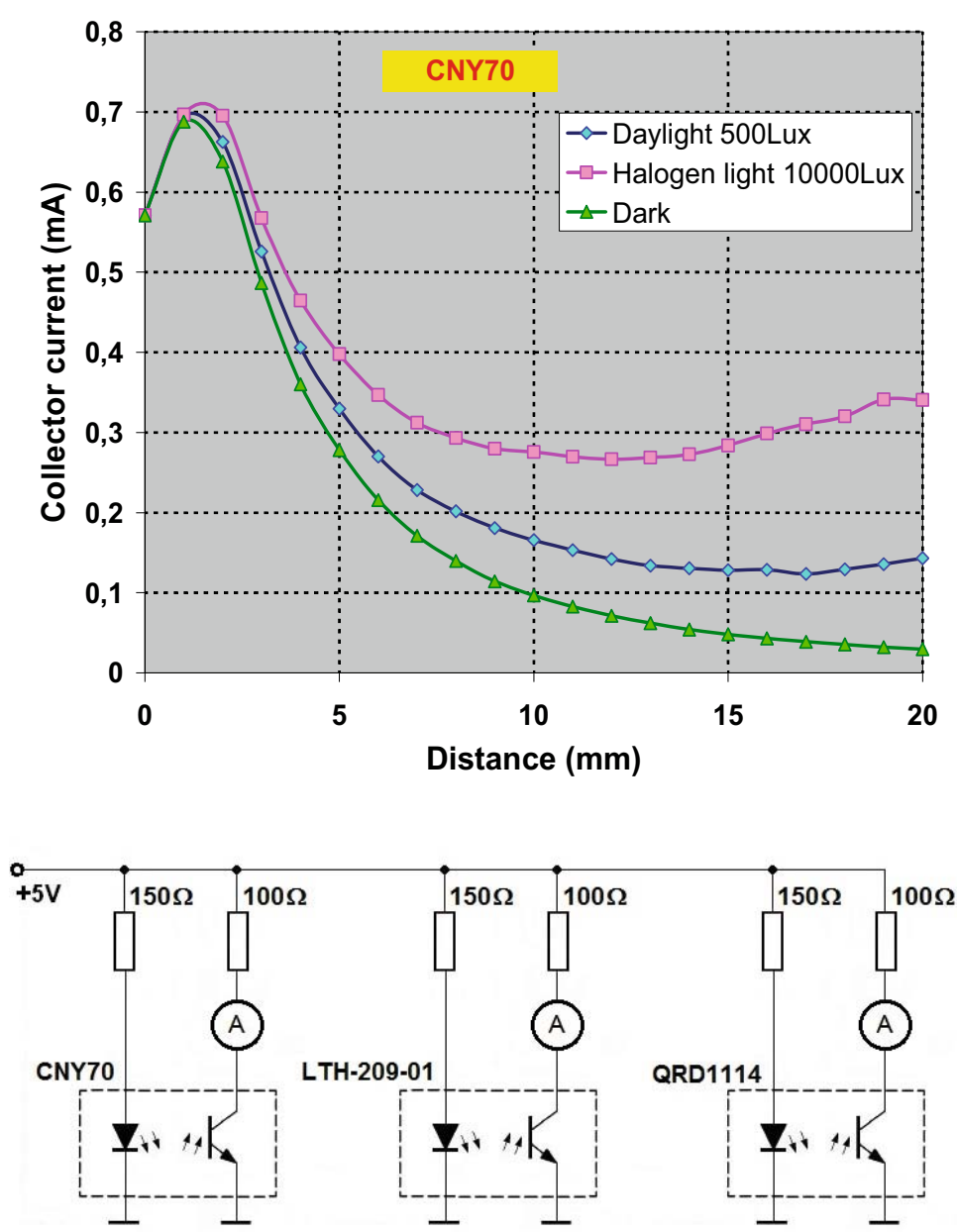

Fig.7. Results of the line recognition sensors testing.

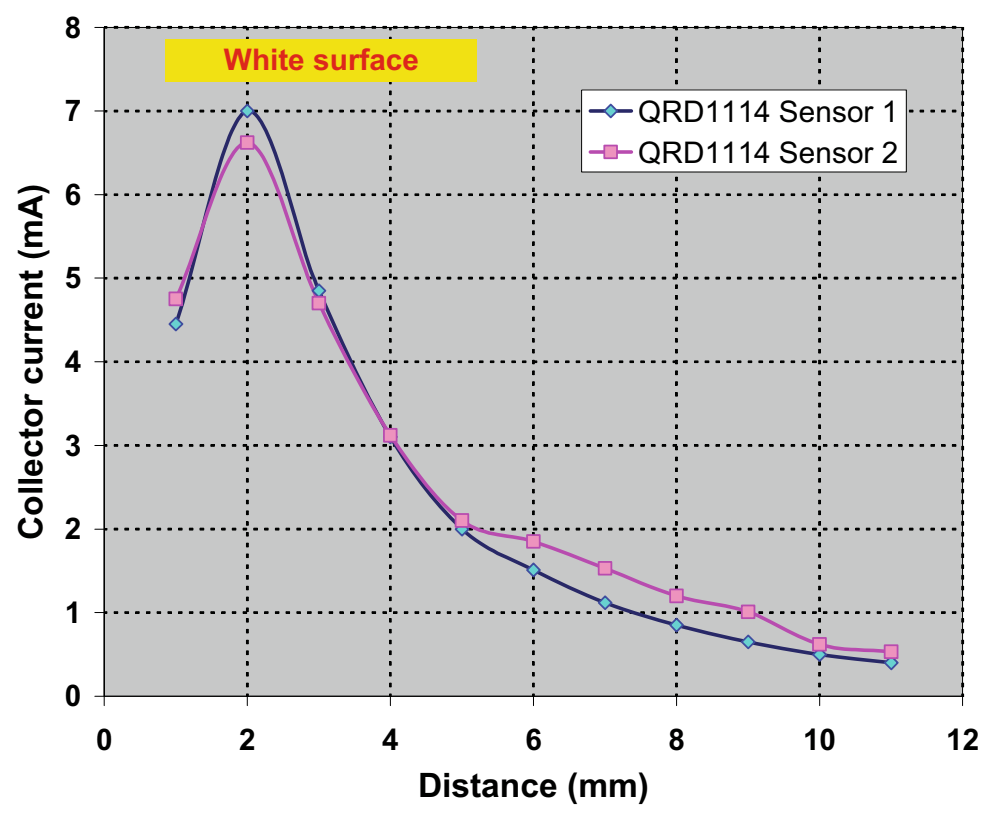




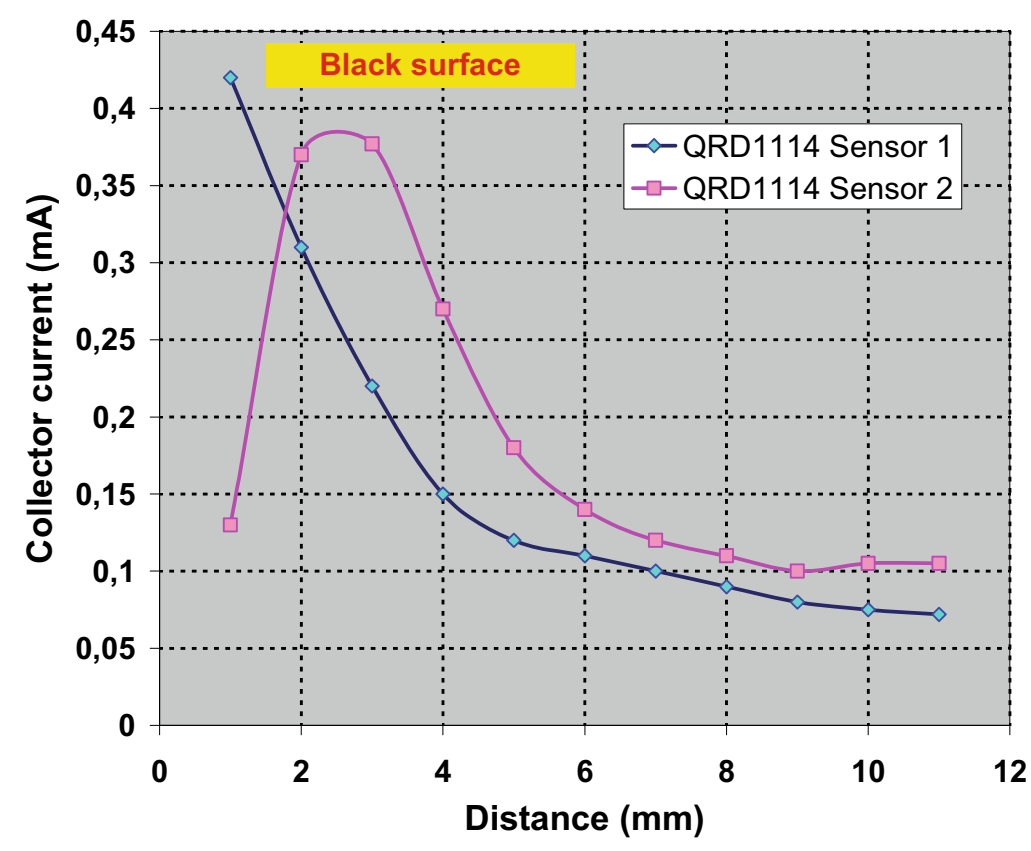

Fig.8. Results of comparative testing of the two samples of QRD 1114.

\subsection{The line recognition sensors issues with ramp}

By moving the sensors ahead of the axis of rotation of the robot a relatively small increase in the positional angle of the robot can be detected with ease, this in turn leads to less radical position adjustments which can cause the robot to loose the line. Instead, it enables small gradual corrections to be made.

The sensors moving in the front brings their own problems regarding clearance with the ramp (Fig.9). If the sensors were moved further in the front of the robot, they would still be required to clear the ramp. A too small distance between the surface and the sensors would result in incorrect feedback, and the robot might enter the ramp at the wrong angle and risk falling off the side (Colville, 2012; Jurisica et al., 2005; Kacir, 2011; Mikova, 2011).
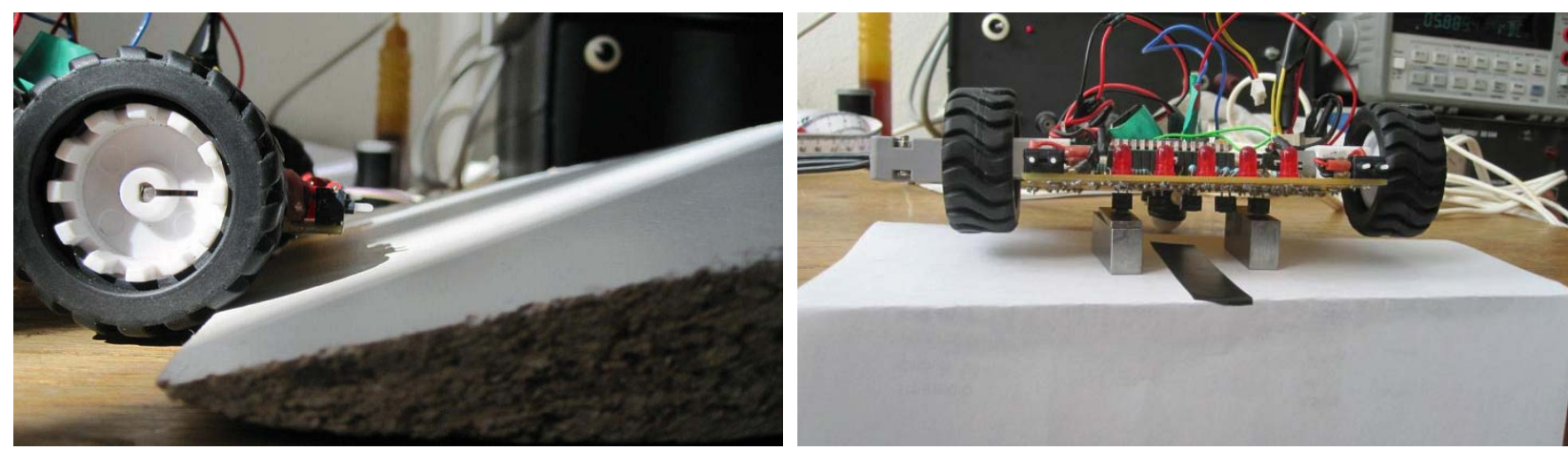

Fig.9. The line recognition sensors issues with the ramp and testing of the line recognition sensor.

So in order to avoid this problem the sensors would be placed higher, which in turn can lead to the problems as the sensor can only register up to a certain distance effectively.

This distance was measured by block gauges, using the SEROUT command of basic atom to measure the value. The measurements (Fig.9) were taken at the 600 Lux light intensity. The values measured 
are determined by the 10 bit resolution $\mathrm{AD}$ converter integrated in the controller. The 5 Volt range is divided into 1024 steps, which equates to $0.004 \mathrm{~V}$ per step.

We decided that the distance of up to $17 \mathrm{~mm}$ between the sensors surface and the reflective surface would be the maximum, as the difference between black and white was becoming smaller and smaller and we would need to be able to tell what was black and white accurately (Fig.10).

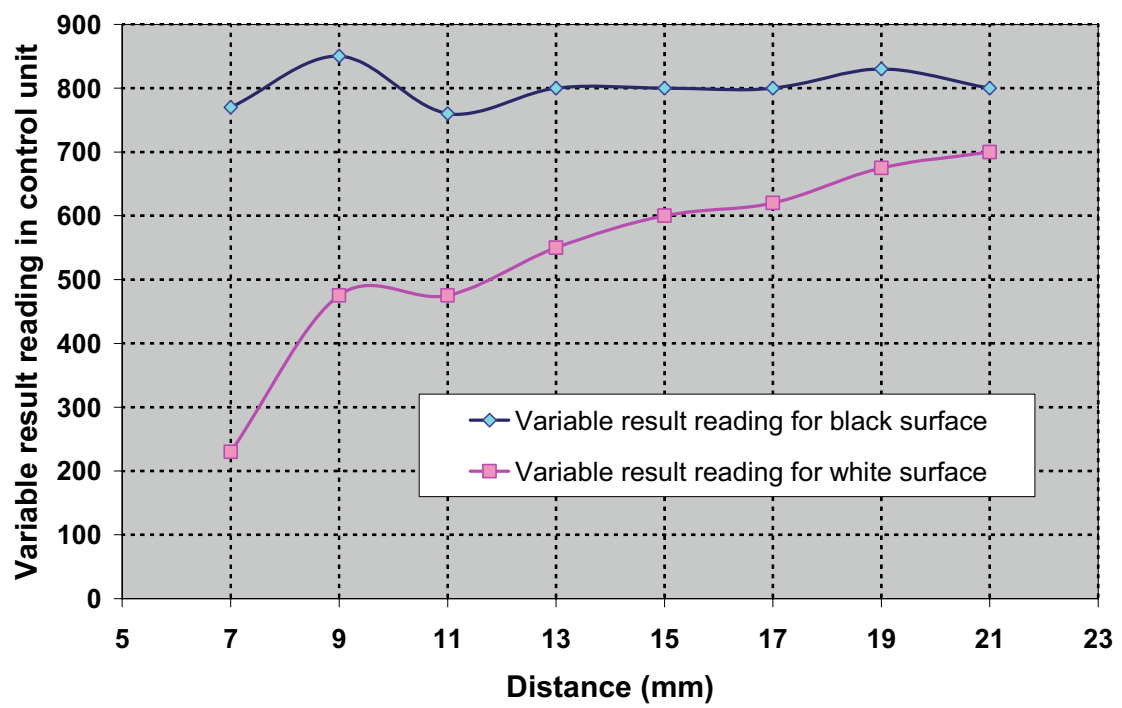

Fig.10. Results of distance measurement of the line recognition sensor with the control unit.
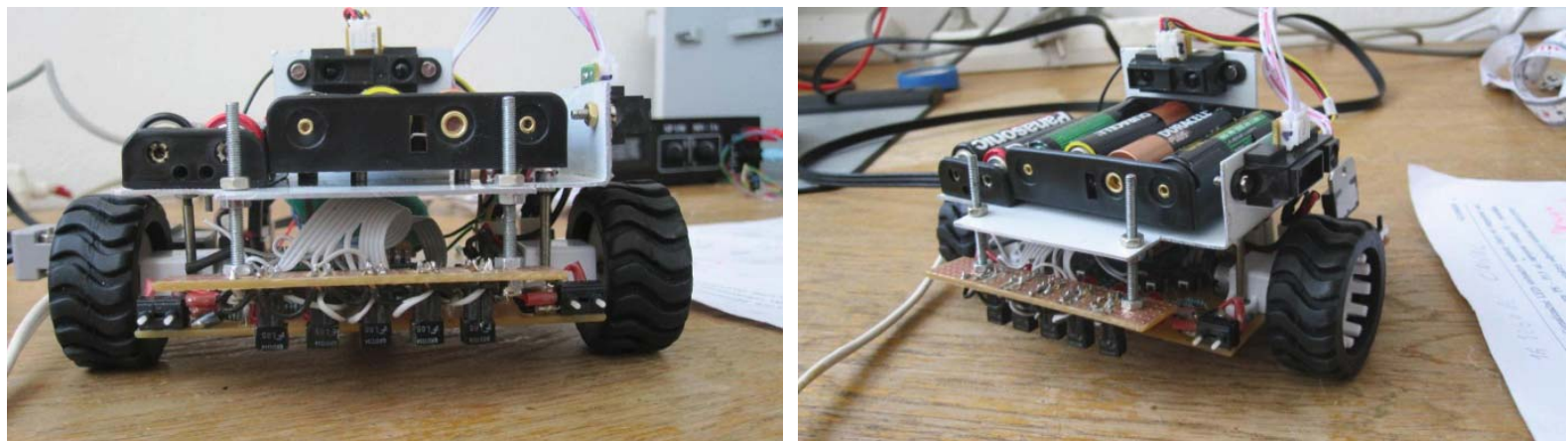

Fig.11. The new position of the line recognition sensors.

Instead of using logical conditions to determine whether or not there was a line under any of the sensors we now required a new set of the conditions consisting of the nominal value under which we could say it was definitely white and the another value we could say it was definitely black. The final calibration is made using the potentiometers for each sensor when the overlap between positively identified white or black points was only 50 points, anything above 650 was definitely black and anything below 600 definitely white.

The new position for the sensors (Fig.11) was hung off the new battery holder bracket, with the option of adjusting height if required, it was set at $15 \mathrm{~mm}$ above the surface to start with.

\section{Testing of the sensor for obstacle detection}

The robot has two Sharp GP2Y0A41SK optical distance sensors for detecting obstacles in its path. Both of the collision sensors were measured (Fig.12) on a range from 0 to $400 \mathrm{~mm}$, with increments of 20 
$m m$, using gauge blocks. Two different surfaces were chosen, red and white. Each measurement was repeated three times, and the average calculated. The measuring method is shown in Fig. 12.
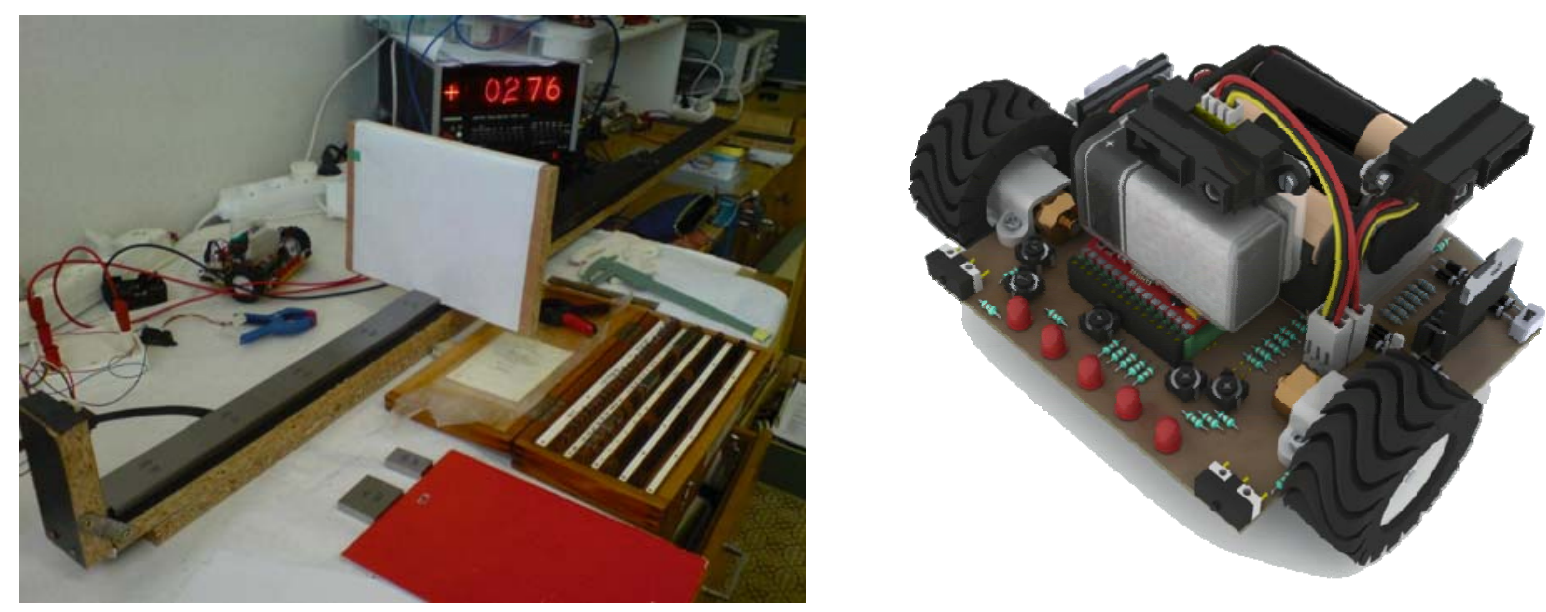

Fig.12. Bench test setup for the Sharp sensors testing and placement of the Sharp sensors on the robot.

From the graph of sensor 1 (Fig.13) we can conclude that a red surface has a larger zone in which the sensor should not be relied upon, as two identical values reported for voltage can have a different distance. The manufacturer states that the minimum measuring distance is $4 \mathrm{~cm}$. It does have the $4 \mathrm{~cm}$ minimum measuring distance as suggested in the data sheet, for both white and red surfaces.

During the experiments on the robot, we observed a problem. The Sharp optical distance sensors were interfering with each other, reporting back false values. This caused hugely inaccurate readings which were unworkable. Both sensors were tested individually and functioned correctly. As a result, we tried to come up with a method to shield one sensor from the other.

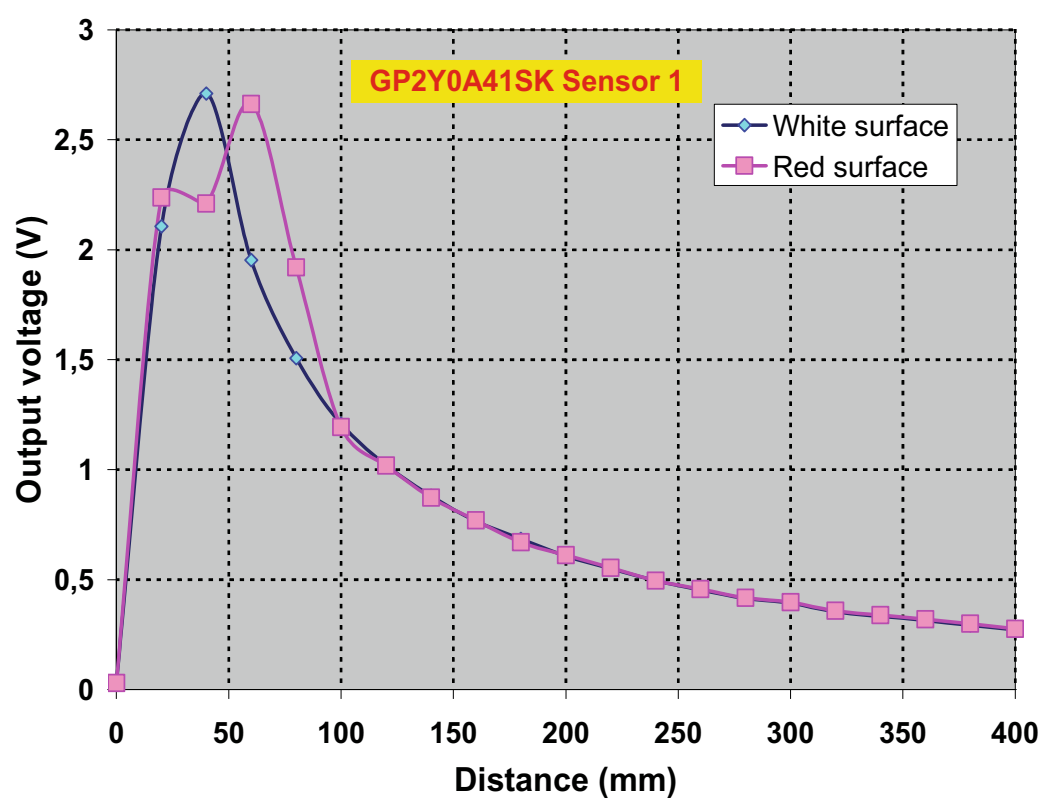




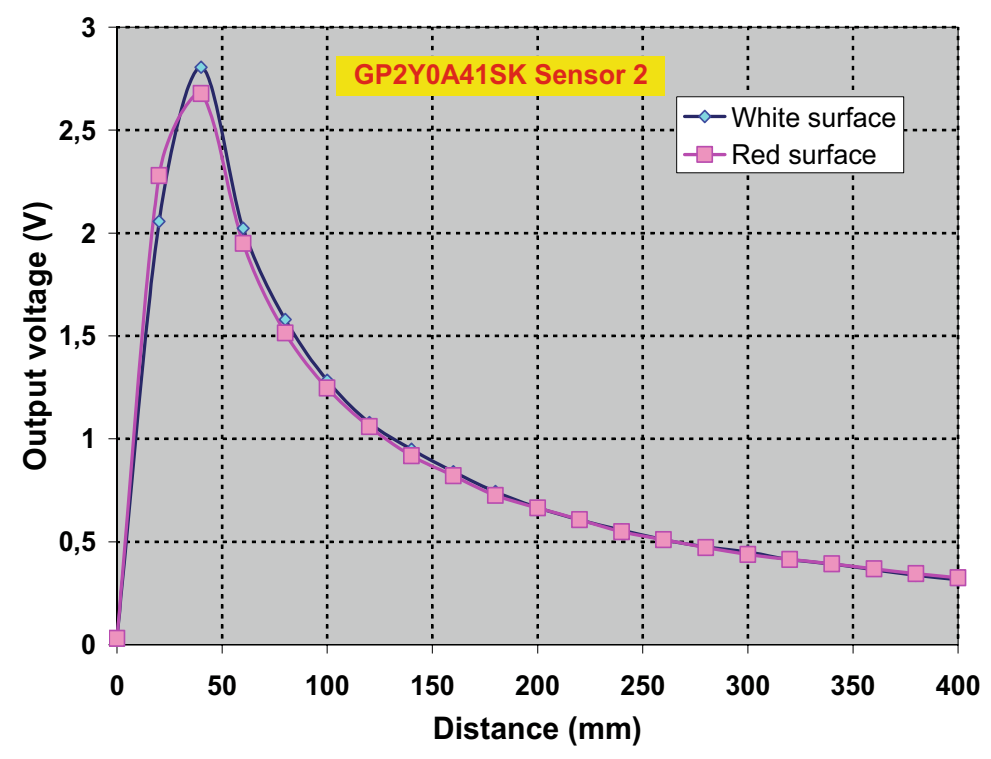

Fig.13. Results of the GP2Y0A41SK optical distance sensors testing.
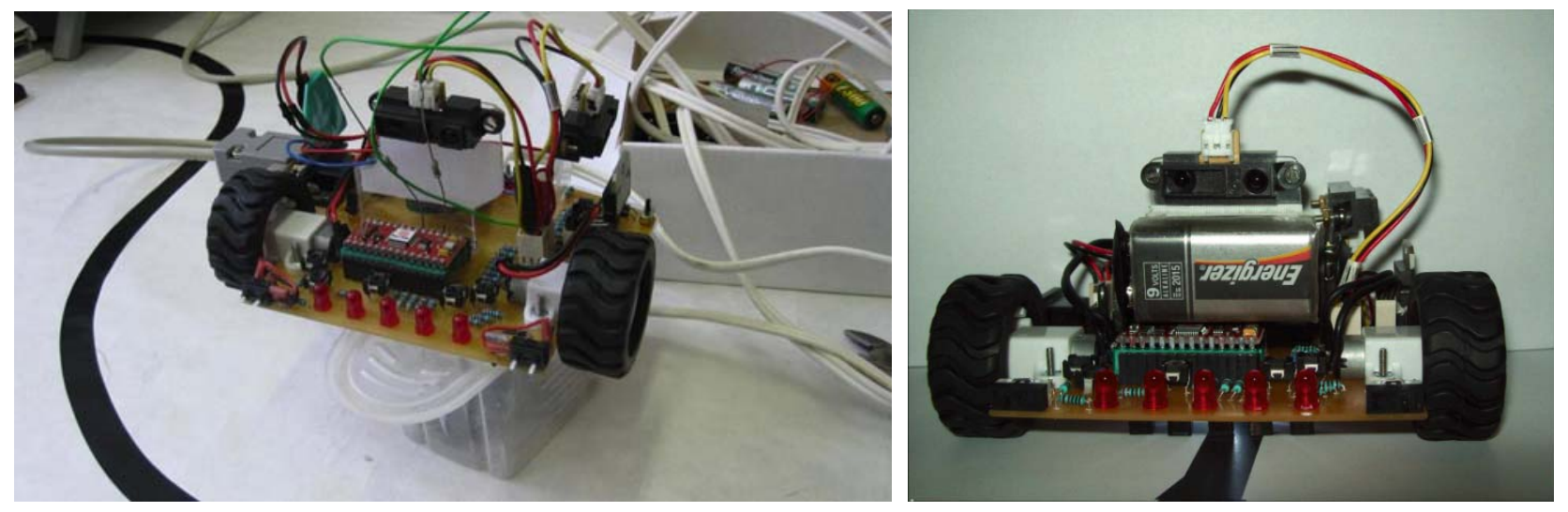

Fig.14. Rebuilding of the GP2Y0A41SK optical distance sensors interfacing to avoid interference.

We tried using a blinder between the two sensors, but in order to function correctly, it would have to be more than $40 \mathrm{~cm}$ long in order to stop reflected IR light bouncing back from the first sensor and being detected by the other sensor. Since this would be completely unpractical, and the robot would be too large to enter the competition, another alternative was required. This problem is solved through the alternation of using these sensors. It means that both sensors are used separately (Fig.14). Activation of both sensors is managed by the control unit.

\section{Conclusion}

The topic of this paper seems to be easy but several key issues arose when working on the prototype of the robot. That is a typical example that also design of a simple product can cause many problems. Practical experience is very important for students. That is something they cannot learn from books. Practical exercises develop their skills and offer experience to the students (Nitulescu, 2008; Vitko et al., 2010). 


\section{Acknowledgement}

The authors would like to thank to Slovak Grant Agency - project APVV-0091-11, VEGA $1 / 1205 / 12$. This contribution is also the result of the project implementation: Centre for Research of Control of Technical, Environmental and Human risks for Permanent Development of Production and Products in Mechanical Engineering (ITMS:26220120060) supported by the Research \& Development Operational Programme funded by the ERDF.

\section{References}

Colville D.J. (2012): Locomotion of the line following robot. - Bs Thesis. Technical University of Kosice, Fac. of Mech. Eng., Kosice, 70 pages.

Jurisica L., Hubinský P. and Kardos J. (2005): Robotics. - Slovak Technical University, Fac. of Elec. Eng., Bratislava. 134 pages.

Kacir K. (2011): Design of functional three wheel vehicle with navigation in unknown environment. - Ms Thesis, Technical University of Kosice, Fac. of Mech. Eng., 2011, Kosice, 62 pages.

Maniarski G. and Kurylo P. (2006): Communication in multiagents systems in robots control. - Theory of Machines and Mechanisms, XX Scientific and Didactic Conference, Zielona Gora, Poland. Press Office University of Zielona Gora, T.1, pp.375-380. ISBN: 83-7481-043-2.

Mikova L. (2011): Simulation model of mechatronics system with four-wheeled lengthwise divided undecarriage with differential control of wheels. - PhD thesis. Technical University of Kosice, Faculty of Mech. Eng. 2011. Kosice. 133 pages.

Nitulescu M. (2008): Experiments in Mobile Robot Control. - Mechatronics 2008. Paper \#200. Available online. Cited 04-07-2013. http://www.mecatronica.pub.ro/romar/articole/mecatronics2008_200.pdf.

Vitko A., Jurisica L., Babinec A., Duchon F. and Klúcik M. (2010): Some Didactic Aspects of Teaching Robotics. - In: AT\&P Journal Plus. ISSN 1336-5010. No.2. Robotics in Education (2010), pp.109-112.

Received: May 20, 2013

Revised: June 14, 2013 